\title{
Crecimiento en niños portadores de insuficiencia renal crónica
}

\author{
Francisco Cano Sch. ${ }^{\text {; }}$ Angela Delucchi B. '; Eduardo Wolff P.'; \\ Eugenio Rodríguez S. ': Angel Fuentes Z. ${ }^{2}$; Teresa Pérez A. ${ }^{3}$
}

\section{Growth in patients with chronje renal failure}

\begin{abstract}
Crie huncred evaluations of weigh/ /eighth and reigh/age were performed in 23 uremic chiltren il under ciatysıs lreatren and 11 che reral transpiantation aged o to 14 yearclc along a seven year follow up. Stavistica

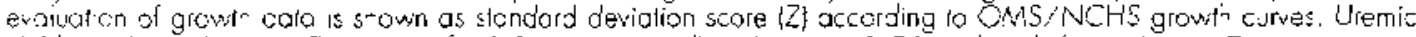
$c^{\text {iild }}=n$ showed mean $Z$ scores of -0.37 in weigtr/heigth ans -2.54 ir heigth/age. Meon $Z$ scores were, correspondingly. 0.29 and -1.89 respectively in children under chronic dyolisis and 0.5 and $-^{\prime} .68$ orer keney iransplantation. Height ve ccily after kidney iransplantction ranged from $28 \mathrm{bl} 151 \%$ when expresed os \% Z score :or the 50 percentile of growth velocily for esch case. No sotiert showed cotch-us growith after kidney transplentation along to menths followns.
\end{abstract}

KKey words: kidney foilure, chron.c. memodialysis, peritoneal diclysis. kidney ronsplantation, growth.)

Entre las consecuencias más comunes y serias de la insuticiencia renal crónica (IRC) se encuentra el retraso del crecimiento que afecta a prácticamente todos los niños urémicos, es tanto más importante cuanto más temprana la edad en que ocurre la ralla renal y más evidente y difícil de recuperar en las dos épocas de la vida en que el crecimiento es rápidamente acelerado: la lactancia y la adolescencia que en la primera depende de factores predominantemente nutricionales $y$ en la segunda está muy influida por las hormonas sexuales ${ }^{1-3}$.

Entre las numerosas causas asociadas a este trastorno del crecimiento destacan factores endógenos, baja ingesta calórica y proteica, trastornos hidroclectrolíticos, desequilibrios de la homeostasia de acidos y bases, anemia, alteraciones precoces $y$ severas del metabolismo del fósforo y el calcio, y alteraciones hormonales.

I. Unidad de Nefrología y Diálisis, Hospital Luis Calvo Mackenna. Departamento de Pediatria Oricnte. Facultad de Medicina, Universidad de Chile.

2. Unidad de Lactantes. Servicio de Pediarría. Hospital Luis Calyo Mackenna.

3. Alumna Cástera de Medicina. Sexto año. División Oriente. Facultad de Medicina, Lniversidad de Chile. como la disminución de la actividad de la insulina o las somatomedinas, cuya producción hepática depende de la hormona del crecimiento y cuyo efector es el cartílago de crecimiento del hueso. La producción de somatomedinas parece estar frenada por una alteración de tos receptores celulares a la hormona de crecimiento, y su acción disminuida por acumulación de proteínas fijadoras plasmáticas o estimulación de factores inhibitorios bajo la influencia de esteroides exógenos ${ }^{3: 4}$. Muchos de estos factores pueden ser modificados y corregidos con un ratamiento oportuno, por lo que la evaluación nutricional periodica debe formar parte del manejo óptimo de estos pacientes. La evaluación nutricional se basa en parámetros clínicos y de laboratorio entre los que destacan la relación peso/talla y talla/edad, mediciones que requieren, no obstante, de una expresión que permita su adecuada utilización clínica, lo cual tradicionalmente se ha realizado mediante los percentiles, fijando el percentil 97 y 3 los límites de la normalidad. Sin embargo, cuando la desviación de la curva va más allá del percentil 3 se requiere otra forma de expresar esta caída para un análisis estadístico de tipo comparativo, por lo cual se ha introducido desde hace algunos años el puntaje 
$Z$ como a método de elección al respecto. El puntaje Z. o puntaje de desviación estándar, se define como la formula:

$$
Z=\frac{X-X_{1}}{D E x}
$$

donde $x$ es el parámero en cucstión, $x_{1}$ es el promedio para la edad y sexo del pacicnte, y DE es la desvíación estándar promedio para dicha edad y sexo. El $Z$ promedio de la población de referencia será 0 . y los márgenes normales que otorgan un intervalo de confianza de $95 \%$ varian entre -2 y +2 DE. correspondiendo a los percentiles 3 y 97 respectivamente. Al usar como referencia la edad y sexo del grupo del paciente para el parámetro estudiado es posible, en forma excepcional, la comparación de poblaciones heterogéncas de modo intraindividual e interindividual mediante esta expresión estadíslical.

Los niños utémicos transitan por tres etapas que ticnen características propias respecto de los ficrores comunes que afectan el crecimiento. La primera, de tratamiento médico, corresponde a zos grados 1 y 2 de la insuficiencia renal crónica ${ }^{6}$; la scgunda es la de diálisis, durante lia cual se agregan elementos propios de este método, como agravación de la anemia, osteodistrofia severa y pérdida de nutrientes por el procedimiento; la tercera es la de trasplante renal que, a pesar dc cstar marcado por un tratamiento jtinumosupresor esteroidal, deberia significar recuperación del crecimiento. Se considera, por lo gencral, que el crecimiento de un niño que ha recibido trasplante renal, es óptimo si la crcatinincmia es inferior a $1 \mathrm{mg} \%$; la dosis de prednisona está cntre 0.12 y $0.18 \mathrm{mg} \cdot \mathrm{kg}$ día en la ctapa de mantención y la cdad ósea, al momento del trasplante, es inferior a 12 años ${ }^{12}$.

Con el propósito de describir la situación del crecimiento de nirios urémicos en nuestro medio, se analizaron las determinaciones antropomé. tricas de un grupo de ellos en las tres etapas de la insuticiencia renal crónica ya mencionadas.

\section{Pacientes y Métodos}

Se analizaron retrospcctivamente las mediciones anturumbétricas para pesoftalla y callatedad en los pacien- tes de insuliciencia renal crónica del Hospital Luis Calyo Mackeuna. de Santiago. Chile, a lo largo de siete años. Los pactentes fueron clasiticados en ires grupos de acuerdo a su función renal. En el grupo I se consideraron 44 mediciones practicadas en 11 enfermos, 8 varones de 6 a 14 años de edad, coll insuficiencia renal crónica grados 1 y 2 bajo tratamento médico. En el segundo grupo $s c$ incluycron 57 evaluaciones realizadas en 17 pacientes ( 9 varones) en tratumiento con dialisis (15 en hemodiadisis, dos en diálisis peritoneal continua ambulatoria) tambjén entre 6 y 14 años de edad. En el grupo 3 se incluyeron 59 mediciones en l] niños que habían recibido trasplante renal (seis varones) de 6 a 17 anos de edad, en los cuales se controlo, además. la velocidad de crecimiento $(\mathrm{cm} / \mathrm{año})$ después del trasplante. La velocidad de crecimiento se expresó en lérminos de la diferencja de $Z$ para el percentil 50 de cada patiense. Todas las evaluaciones nutricionales fucron realizadas por un médico pediatra especialista en nulrición. El peso y la estatura de cada paciente fueron convertidos a unidades $Z$ de acuerdo con las tablas de peso paria talla $y$ talla para edad de la Organization Mundial de la Salud (OMS) y el Centro Estadounidense de Estadísticas de Silud (NCHSi. De las citrus obtenidas se calcularon los promedios para cada uno de los grupos descritos. Debido a la amplia variabilidad de cada disıribución no se aplicaron prucbas $t$ de Student. Se efectuó análisis de varianza simple ANOVA que no mostó diferencias significativas entre les grupos

\section{Resultados}

En el grupo 1 los promedios de $Z$ para pesol talla y talla/cdad fueron $-0,37$ y $-2,54$ respectivamente. En los pacientes sometidos a diálisis el promedio de $Z$ fue 0,29 para pesoltalla y $-1,89$ para talla/edad. En niños que habían recibido trasplantes el promedio de $Z$ fue 0,5 para pesol talla y de - 1,68 para talla/edad. Los grupos no mostraron diferencias estadísticamente significativas entre ellos cuando el crecimiento se midió en forma comparativa para el puntaje de desviación estándar, pero $\mathrm{Z}$ fue más cercano a la normalidad en pesoltalla, mientras que mostró serio deterioro en talla/edad en todos los grupos (tabla 1).

La velocidad de crecimiento en los niños que habian tecibido trasplantes renales en un período comprendido entre los 18 meses anteriores y 66 meses después alrededor de la fecha de la

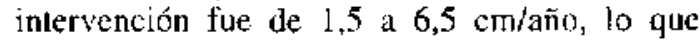
corresponde a un nargen de cambio de 28 a $151 \%$ con respecto a la mediana de $Z$ para los 11 pacicntes (tabla 2). La diferencia del puntaje $\mathrm{Z}$ en ocho de estos niños osciló entre $-0,03$ y +0.49 (tabla 2 ). En ningún paciente sometido a trasplante se obtuvo una diferencia que sugirie- 
Tabla 1

Rejación peso/talla y talla/edad en pacientes con insuficiencia renal en tratamiento médico sin diálisis. sometidos a diálisis y después de trasplante renal

\begin{tabular}{|c|c|c|c|}
\hline & $\begin{array}{c}\text { IRC } \\
\text { Sin diálisis } \\
Z=D E\end{array}$ & $\begin{array}{c}\text { IRC } \\
\text { En diálisisis } \\
\mathbf{Z} \pm \mathrm{DE}\end{array}$ & $\begin{array}{c}\text { IRC } \\
\text { Post trasplante } \\
\text { Z } \pm \text { DE }\end{array}$ \\
\hline $\begin{array}{l}\text { Peso/talla } \\
\text { Talla/edad }\end{array}$ & $\begin{array}{l}-0.37 \pm 1.37 \\
-2.54 \pm 1.26\end{array}$ & $\begin{array}{r}0,29 \pm 0,95 \\
-1.89=1,38\end{array}$ & $\begin{array}{r}0.50 \pm 1.95 \\
-1.68 \pm 1.12\end{array}$ \\
\hline $\begin{array}{l}\text { Alowil peso } \\
\text { Alowa talla }\end{array}$ & $\begin{array}{l}\text { la } F: 1.4] \mathrm{ns} \\
\text { ad } F: 2.54 \mathrm{~ns}\end{array}$ & & \\
\hline
\end{tabular}

IRC: insuficiencia renal crónica

se crecimiento rápidamente acelcrado y no se observó correlación positiva entre la velocidad de crcuimicnto después de csta intervención y la edad del paciente al momento de hacerla.

\section{Comentario}

Los resulados expuestos son similares a los de otras experiencias ${ }^{7.8}$ c indican que el retraso de crecimiento del paciente urémico se proyecta aún más allá del trasplante renal. Esto obliga a revisar cada factor involucrado y hacer un esfuerzo para corregirlo. idealmente antes de su expresión elínica ${ }^{2} .9 .10$. La escasa relación entre la velocidad de crecimiento (delta $Z$ ) y la edad al momento del trasplante pudiese significar que el ticmpo durante el cual actuaron los diferentes factores involucrados en el retraso de talla fue suficientemente largo como para sobrepasar el efecto de una bucna función renal en el período posterior al trasplantc. Este argumento ha influido en la decisión de hacer trasplante a pacientes que todavía mantienen cierto grado de filtración glomerular, pero aún no han entrado a la etapa donde se requiere diálisis ${ }^{14}$. El único paciente de esta serie que recibió un trasplante antes de requerir dialisis es el que ostenta el porcentaje de velocidad de crecimiento más alto (151\%). En seguimientos de pacientes hasta por 10 años después del trasplante se ha observado que la desaceleración de la talta puede incluso cmpeorar ". En grupos más numerosos el crecimiento acelerado sólo fue alcanzado por pacientes menores de un año al momento del trasplante, to que resalta quc, pese a todo, la edad del trasplante es un factor importante en el crecimiento 15 .

Es bien conocido el efecto deletéreo de los corticoides en el crecimiento, por inhibición de la secreción de hormona del crecimiento ${ }^{16}$, acción sobre la aclividad biológica o estimulación de inhibidores de sus mediadores, las somatomedinas o factores de crecimiento tipo insulina. y también por efecto local en el metabolismo oseo, donde interfieren con el crecimiento del cartílago y la mineralización del hueso. Ha sido posible disminuir o evitar su uso en los tratamientos inmunosupresores que siguen al trasplante renal, especialmente despućs de la intro-

Tabla 2

Velocidad de crecimiento en once nin̄os con insuficiencia renal crónica sometidos a trasplante renal

\begin{tabular}{|c|c|c|c|c|c|}
\hline $\begin{array}{l}\text { Pacientr } \\
\text { (sexul) }\end{array}$ & $\begin{array}{l}\text { Edad } \\
\text { (años) }\end{array}$ & $\begin{array}{l}\text { Velocidad de } \\
\text { crecimiento } \\
\text { (cm/año) }\end{array}$ & Mediana & $\%$ del p50 & Delta $\mathbf{Z}$ \\
\hline$]_{(\mathrm{m})}$ & 6 & 5.0 & 6.2 & 80,64 & 0,49 \\
\hline $2(110)$ & 10 & 3.0 & 4,8 & 62,50 & $-0,20$ \\
\hline $3(t)$ & 11 & .3 .5 & 4.7 & 74,46 & $-0,38$ \\
\hline $4(1)$ & 11 & 6.0 & 4.8 & 125.00 & - \\
\hline 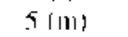 & 12 & 6.5 & 4,3 & $15 \mathrm{~J}, 16$ & - \\
\hline $6(0)$ & 12 & 1,5 & 5.3 & 28,30 & $-0,03$ \\
\hline $7 \mathrm{~cm})$ & 13 & $3,1)$ & 4,5 & 66,66 & 0,14 \\
\hline $8(\mathrm{~m})$ & 13 & 5.5 & 4,5 & 122,22 & - \\
\hline $4(f)$ & 13 & 2,5 & 4,5 & 55,55 & 0,43 \\
\hline $10 \mathrm{im}$ & 14 & 6,5 & 5,8 & 112,06 & 0.285 \\
\hline 11 (m) & 14 & 3,5 & 6.0 & 58,33 & -0.11 \\
\hline
\end{tabular}

m: sexo masculino

f: sexo femenimo 
ducción de la ciclosporina. Todo intento en este sentido debe asumir cuidadosamente el riesgo de rechazo del injerto, que en algunas experiencias ha alcanzado proporciones de hasta $40 \%$ en niños en los cuales se discontinuó la prednisona seis meses después del trasplante. Sin embargo. cstudios multicéntricos de otros países, con más de 20 mil pacientes sometidos a trasplante, sugieren que la sobrevida del injerto a cinco años puede ser aún superior sin prednisona y que puede obtenerse crecimiento adecuado en niños sometidos a trasplante al suspender los esteroides ${ }^{9}$, si bien el crecimiento rápidamente acelerado se registró sólo en niños prepúberes ${ }^{4.9}$. Puesto que la suspensión de los esteroides en niños con trasplante renal parece asociarse con elevado número de rechazos agudos ${ }^{12.15}$, se han realizado intentos de emplear prednisona en días alternos, con buenos resultados expresados en un cambio favorable del crecimiento, sin rechazo, al cabo de un año ${ }^{17}$. En nuestra unidad, hay cuatro pacientes manejados con este esquema, que no han sufrido rechazo agudo en seguimientos de 24 a 36 meses.

\section{Resumen}

Se hicieron 160 determinaciones de peso/talla $y$ talla/edad en 28 pacientes portadores de insuficiencia renal crónica (IRC), 17 de ellos en diálisis y 11 sometidos a trasplante renal, de 6 a 14 años de edad, en un período de 7 años. Los resultados se expresan como puntaje de desviación estándar (Z). En los niños con insuficiencia renal crónica el promedio de $Z$ fue $-0,37$ y $-2,54$, respectivamente, para peso/talla y talla edad. En los enfermos en tratamiento con diálisis los correspondientes promedios fueron 0,29 y $-1,89$ y en los niños que habían recibido trasplantes renales 0,5 y $-1,68$ respectivaniente. En estos últimos pacientes la velocidad de crecimiento después del trasplante osciló entre 28 y $151 \%$ del percentil 50 para cada caso y ninguno de ellos mostró crecimiento rápidamente acelerado. Los factores involucrados en el retardo del crecimiento del niño urémico antes y después del transplante renal se analizan en detalle, con especial énfasis en el rol que juegan los esteroides suprarrenales en estos últimos.

(Palabras clave: insuficiencia renal crónica, diálisis peritoneal, hemodiálisis, trasplante renal, crecimiento.)

\section{Referencias}

I. Mehis $O$, Schaefer F, Tonshoff $B$ : Patterns of growth in children with chronic renal failure. In: Growth Disorders in Chronic Renal Failure, Ed. by Mehis O., Oxford Clinical Communications, Crown House 1991; $1-3$.

2. Heimburger $O$, Bergstrom J, Lindholn B: Maintenance of optimal netrition in CAPD. Kidney Int 1994; 46: 39-46.

3. Fine R. Stablein D, Tejani A: Do children exhibit catch-up growth post transplantation: North American Pediatric Renal Transplantation Cooperative Study. Pediatr Nephrol 1995; 9: s66-s68.

4. Mehis $O$, Schaefer $F$, Tonshoff B: Patterns of growth in children with chronic renal failure. In: Growth Disorders in Chronic Renal Failure, Ed, by Mehis O., Oxford Clidical Communications, Crown House 1991; 9-27.

5. Abitol $C$, Zillemeto $G$. Srrauss $J$ : Assessment of growh and nutritional requeriments in uremic children. In-Inmunosuppression and renal disorders in neonates and children, ed. by Strauss J., University of Miami Press, Coral Gables, Florida 1989: 1-10.

6. Delucchi A. Cano F: Insuficiencis renal crónica. Rev Chil Pediatr 1990; 61: 19-20.

7. Rees L: Growth posttransplantation in children: sterojds and growth inhibition. In: Pediatric Renal Transplantation, ed. Wiley-Liss lnc. 1994: 423-439.

8. Mehis O, Schefer $F$. Tonshoff B: Pattems of growth in children with chronic renal failure. In: Growth Disorders in Chronic Renal Failure, Ed. by Mehis O.. Oxford Clinical Communications, Crown House 1991: 33-40).

9. Ingulli E. Tejani A: Steroid withdrawal aftef renal transplanfation. In: Pediatric Renal Transplantation. ed. Wiley-Liss Inc. 1994: 221-238.

10. Cuno $F$ : Osteodistrofia renal en el niño. Rev Chil Pediatr 1992: 63; 166-172.

I1. Putter D. Feduska $N$, Melzer J, et al: Twenty years of renal transplantation in children. Pediatrics 1986: 77: 46.5-470.

12. Fine $R$, NeIson $P$, Enenger $R$ : Growth following renal transplantation in children. In: Inmunosuppression and renal disorders in neonates and children. ed. by Strauss J., University of Miami Press, Coral Gables, Flotida 1989; 17-27.

13. Kaiser B. Baluttrte $H$ : Growth in children undergoing long term dialysis. In: Inmunosuppression and renal disorders in meonates and children, ed. by Struuss J., Universily of Miami Press, Coral Gables, Florida $1989 ; 17-27$.

14. Levey A. Milford E: Donor and recipient selection. In: Renal toansplantation, ed. by Milford E. Brenner B. Stein J, Churchill Livingstone, New York, Edinburgh, London. Melbourne 1989: 247-281.

15. McEnery P. Stablein D. Arbus G. Tejant A: Renal rransplantation in children: a report of the North Ametican pediatric renal transplant cooperative study. N Engl J Med 1992; 326: 1727-1732.

16. Jabs $K$, Yan Dop C. Harmon W: Endocrinologic evaluation of children who growth poorly following renal transplantation. Transplandation 1990; 49:71-76.

17. Brover M, Guest G. Gagnadom M: Growth rate in children receiving atemate day corticosteroid treatment after kidney Iransplantation. J Pediatr 1992: 120: 72 1-725. 